\title{
Condiciones institucionales para el ejercicio académico de la investigación de la comunicación en México
}

\author{
Raúl Fuentes Navarro \\ Departamento de Comunicación, ITESO. \\ DECS, Universidad de Guadalajara
}

Este trabajo tiene por objeto analizar tanto documental como empíricamente las condiciones institucionales bajo las cuales se practica la investigación académica de la comunicación en México desde una perspectiva histórica y al mismo tiempo estructural. Es decir, se tratan de recuperar, primero, los factores que han determinado su institucionalización para caracterizar el estado actual y, a partir de ese diagnóstico, proyectar sus tendencias para el futuro inmediato, dentro del contexto económico, político y cultural general del país y del sistema nacional de educación superior.

Este análisis de las condiciones institucionales para la práctica académica de la investigación se ubica como parte de un proyecto de pretensiones más amplias, que el autor realiza como tesis de doctorado, ${ }^{1}$ y en el cual se concibe a la institucionalización de un campo académico como un proceso desarrollado en dos planos principales: el cognoscitivo y el social. Aunque este trabajo se refiere a sólo una de las dimensiones del segundo de estos planos, la organización institucional de los recursos y programas académicos, su análisis es complejo, pues no puede desvincularse del de otras dimensiones de este plano, como son las publicaciones especializadas y las asociaciones académicas, ni de sus articulaciones con las dimensiones del plano de la institucionalización cognoscitiva.

1 Se trata del proyecto Determinaciones Socioculturales del Campo Académico de la Comunicación en México, en proceso dentro del programa de Doctorado en Ciencias Sociales (Area de Sociología) ofrecido desde 1991 por la Universidad de Guadalajara y el Centro de Investigaciones y Estudios Superiores en Antropología Social (Ciesas). El autor agradece los apoyos recibidos para la realización de este proyecto por parte del ITESO y del Seminario de Estudios de la Cultura del Consejo Nacional para la Cultura y las Artes. 
En la organización social del campo académico, cuyas condiciones específicas se exploran aquí para el caso del estudio de la comunicación en México, se sitúan las prácticas por medio de las cuales los sujetos adquieren y refuerzan su identidad como "investigadores" al mismo tiempo que concretan su profesionalización y contribuyen a la estructuración del propio campo. De esta manera, la institucionalización del campo se postula como un proceso inseparable de la profesionalización y de la legitimación de las prácticas y sus productos.

\section{El estudio de la comunicación como práctica científica}

A pesar de que los programas de docencia en comunicación comenzaron a institucionalizarse en México desde los años cuarenta, fue hasta los sesenta cuando comenzaron a realizarse prácticas (aisladas) de investigación y en los setenta cuando se dieron los primeros intentos de institucionalización de esta actividad, tanto dentro como fuera de los establecimientos universitarios. Como diagnóstico pionero de este desarrollo, en marzo de 1974, Josep Rota presentaba el siguiente balance:

Durante los últimos diez años, la mayor parte de la investigación ha sido comercial, realizada por agencias de publicidad o compañías de investigación de mercados. Desgraciadamente, los resultados de estos esfuerzos suelen ser confidenciales. Casi la totalidad de la investigación está constituida por las tesis de licenciatura de estudiantes universitarios, sobre todo del Departamento de Comunicación de la Universidad Iberoamericana. Se han escrito ahí 43 tesis entre 1967 y 1973. Otras se han realizado en la Universidad Nacional Autónoma de México. Pero aparte de las tesis, prácticamente no se ha hecho nada más (Rota, 1974: 56).

Para 1980, según el Análisis de la situación actual de la investigación empirica de la contunicación en México coordinado por Rubén Jara, realizado sobre 100 estudios,

Los resultados de la revisión efectuada son claramente indicativos de que no existen actualmente en México las condiciones adecuadas para que se realice de manera apropiada una labor de investigación en comunicación: 
a) No hay claridad sobre las prioridades de investigación;

b) No hay acuerdo sobre las orientaciones teóricas o metodologicas que debieran seguirse;

c) No hay una infraestructura sólida de apoyo económico y técnico para las investigaciones universitarias, las cuales representan la actividad prioritaria de investigacion;

d) No existen investigadores capacitados dedicados primordialmente a la labor de investigación. Estos más bien son pasantes de licenciatura o profesores universitarios que se dedican fundamentalmente a la docencia;

e) Cuando a pesar de todos los obstáculos se realizan trabajos valiosos, éstos tienen una circulación muy restringida debido a la falta de medios y sistemas de intercambio de información adecuados (Jara, 1981: 214).

Las conclusiones de Rota y Jara, en sus respectivas revisiones del "estado de la cuestión", pueden tomarse como base de diagnósticos más recientes sobre la investigación de la comunicación en México desde sus condiciones, como el realizado en este trabajo. Ambos indican, antes que nada, la severa limitación de las infraestructuras indispensables para la práctica de la investigación en las universidades mexicanas. Josep Rota y Rubén Jara fueron los dos primeros mexicanos becados para estudiar un doctorado en Comunicación en los Estados Unidos, según un convenio establecido entre la Universidad Iberoamericana y la Universidad Estatal de Michigan en 1970, que a su regreso al país tuvieron la encomienda de impulsar la investigación académica. Sin embargo, los "centros" fundados por Rota primero en la Universidad Iberoamericana en 1974 (después encargado a Jara), y luego en la Universidad Anáhuac en 1975, no alcanzaron la solidez institucional suficiente para perdurar en los ochenta ${ }^{2}$ (Véase Fuentes, 1991; 1993).

En términos más amplios, aunque específicamente relacionados con las condiciones institucionales, cabe mencionar que en 1984 el Consejo Mexicano de Ciencias Sociales (Comecso) y el Consejo Nacional de Ciencia y Tecnología (Conacyt) levantaron una encuesta en 390 centros de investigación en ciencias sociales en todo el país, incluyendo seis dedicados al estudio de la comunicación.

2 Ambos investigadores fueron entrevistados en 1993 por el autor para este proyecto, y reconstruyeron tanto las experiencias que enfrentaron entonces, como sus apreciaciones actuales, habiéndose ambos alejado de las universidades mexicanas a principios de los ochenta, sin dejar de practicar la investigación: Rota trabaja en la Universidad de Ohio, en los Estados Unidos, y Jara dirige una importante agencia de investigación aplicada en la ciudad de México. 
De éstos, sólo uno ofrecía "las condiciones mínimas para el desarrollo de sus tareas y otros dos podrian alcanzarlas en el corto plazo". Estos tres centros se situaban en la ciudad de México. Los tres restantes (uno en la capital y dos fuera), no parecían tener esperanzas de llegar a satisfacer los requerimientos básicos para realizar investigación científica, según los criterios de la encuesta. Entre las conclusiones generales de este diagnóstico vale la pena citar la siguiente:

Estos resultados muestran una estructura institucional de la investigación científica en ciencias sociales con grandes deficiencias estructurales, o vicios que se han acumulado, de tal magnitud, que se constituyen en un lastre difícil de eliminar y una de las más grandes barreras al desarrollo de la investigación cientifica. De all que la formación de recursos humanos al nivel de posgrado es una de las tareas de mayor prioridad (Benítez, 1987: 52).

Es evidente que hay una relación muy estrecha entre la investigación y la formación de investigadores en los posgrados universitarios. Pero este nivel, según Brunner, "es todavía en extremo precario en América Latina":

El cuarto nivel; o nivel de posgrado, se halla relativamente institucionalizado sólo en dos países de América Latina: Brasil y México. Pero incluso allí, y de manera más marcada en los demás países de la región, se observa que el desarrollo de ese nivel es profundamente desigual y heterogéneo. Sólo una proporción de los programas de posgrado, variable según el país, sirve para la formación de investigadores y esto no siempre ocurre a nivel de doctorado. Con la excepción de Brasil, se constata en varios casos que los programas de posgradoson atendidos por profesores que no hanalcanzado la más alta calificación académica, que no realizan continuamente investigación y que no publican sistemáticamente bajo formas reconocidas por sus pares. Una alta proporción de los docentes de estos programas son relativamente jóvenes, en tanto que sus alumnos, también con la excepción de Brasil, carecen en muchos casos del apoyo de becas, de acceso a bibliotecas y equipos adecuados y, en proporciones variables, trabajan junto con estudiar (Brunner, 1990: 158).

Aunque la formación en los posgrados en comunicación mexicanos no es objeto de atención de este trabajo, esta apreciación puede resumir algunas de las condiciones imperantes para la práctica de la investigación en México, pues describe muy bien los límites con los que se trabaja.

Por otra parte, aunque la Asociación Mexicana de Investigadores de la Comunicación (AMIC) se propuso desde 1980 como una de sus prioridades "diagnosticar 
el estado actual" de la investigación, fue hasta su IV Reunión Nacional (Guadalajara, febrero de 1987) cuando algunos de sus miembros abordaron la tarea. De ahí surgieron como "obras gemelas" un libro compilado por Enrique Sánchez Ruiz (1988) y la Sistematización Documental 1956-1986 de Raúl Fuentes Navarro (1988) sobre la investigación mexicana de la comunicación.

Un año después, ambos autores introdujeron la figura de la triple marginalidad de la investigación de la comunicación (con respecto a las ciencias sociales, de éstas en el conjunto de la actividad científica y de ésta en relación con las prioridades del desarrollo nacional) (Fuentes y Sánchez, 1989) y continuaron actualizando el análisis del campo en algunas colaboraciones conjuntas. Una de ellas caracteriza al periodo 1985-1990 como "de transición" para la investigación mexicana de la comunicación, incluyendo su estructura institucional de base:

Hasta 1985, prácticamente la totalidad de la investigación mexicana de la comunicación se realizó en la ciudad de México, ya fuera en centros universitarios o de otro carácter. La investigación académica estuvo mayoritariamente concentrada en la Universidad Nacional Autónoma de México (UNAM), aunque con importantes complementos en la Universidad Autónoma Metropolitana (UAM-X), la Universidad Iberoamericana (Uia) y, durante unos años, la Universidad Anáhuac. La investigación no universitaria ha incluido centros privados, como Comunicología Aplicada de México (del grupo publicitarioFerrer) y el Institutode Investigación de la Comunicación (filial de Televisa); otros internacionales, como el Instituto Latinoamericano de Estudios Transnacionales (ILET), el Instituto Latinoamericano para la Comunicación Educativa (ILCE) yel Centro de Estudios Económicos y Sociales del Tercer Mundo (Ceestem); se pueden incluir también algunos centros paraestatales como el Centro Nacional de Productividad (Cenapro)y el Centro de Mediosy Procedimientos Avanzados de Educación (Cempae) y diversas dependencias del gobierno federal que, especialmente en los anos setenta, contribuyeron de manera importante en diversas áreas del estudio de la comunicación. La crisis provocó que la mayor parte de estos centros, ubicados todos en la capital del país, disminuyeran considerablemente su producción, o cerraran (Fuentes y Sánchez, 1992: 25).

Debido a lo que globalmente se conoció como "la crisis" de los ochenta, hasta 1990, según esa figura de "transición", la proporción de la investigación realizada en la UNAM se redujo drásticamente, mientras que la de la UAM-X se incrementó un poco; la de la Uia se sostuvo, pero la aportación de la Universidad Anáhuac se retrajo mucho, así como las de Comunicología Aplicada e ILET. Finalmente, el Ceestem, así como los centros paraestatales (Cenapro y Cempae) y los formados 
en varias secretarias de estadoy dependencias oficiales fueron víctimas, en diversos momentos, de los recortes presupuestales del gobierno federal y desaparecieron.

No obstante, en el mismo periodo, se crearon nuevos centros de investigación de la comunicación en el país y se incrementaron los espacios de diálogo e interrelación tanto entre instituciones como entre investigadores, a través de reuniones de trabajo, proyectos específicos y publicaciones periódicas. Entre los nuevos centros, que incorporaron a investigadores posgraduados tanto en el extranjero como en el país, e impulsaron la investigación de manera muy notable en la segunda mitad de los ochenta, destacan tres principales: el Centro de Estudios de la Información y la Comunicación (CEIC, hoy Departamento de Estudios de la Comunicación Social) de la Universidad de Guadalajara, el Programa Cultura de la Universidad de Colima y el Programa Institucional de Investigación en Comunicación y Prácticas Sociales (Proiicom) de la Universidad Iberoamericana, sólo éste último asentado en la capital (Fuentes y Sánchez, 1992: 26-27).

Junto a algunos de los programas de posgrado, estos tres centros de investigación se han constituido, en los últimos diez años, en el núcleo de una práctica de la investigación de la comunicación quizá por primera vez verdaderamente sistemática, interdisciplinaria, colectiva y nacional, relativamente independiente de los programas de licenciatura y con proyección al menos iberoamericana. En algún sentido, la crisis económica de los ochenta, al mismo tiempo que desestructuró la configuración que el campo había adquirido en los setenta, propició una reestructuración aparentemente más solida institucionalmente y más productiva académicamente, que cabe analizar con mayor detalle.

En primer lugar, se detecta una tendencia clara hacia el distanciamiento entre la investigación "aplicada" o "comercial" y la académica. Los proyectos más directamente vinculados con la toma de decisiones en algunos ámbitos de las prácticas sociales de comunicación, que los norteamericanos llaman "investigación administrativa", se desplazaron decididamente hacia agencias especializadas, siguiendo el auge de los estudios de mercado y de opinión pública que trajo consigo la "modernización" económica y el "adelgazamiento" del Estado.

Por su parte, la mayoría de los proyectos académicos se concentraron en la profundización - crítica - del conocimiento sobre diversas temáticas y desde distintos enfoques metodológicos - predominantemente "cualitativos" - , aunque paradójicamente incrementaron su grado de desvinculación con la formación profesional de los estudiantes de comunicación. Con la excepción del "reforza- 
miento" de algunos programas de posgrado, la investigación académica encontró nuevos espacios de desarrollo mediante relaciones más estrechas con centros, investigadores y enfoques de otras disciplinas de las ciencias sociales que con las licenciaturas en comunicación. Es muy elocuente en este sentido la orientación de los tres centros creados en los ochenta, totalmente desvinculados de las carreras profesionales, y en los cuales se ha concentrado la producción de investigaciones y publicaciones en los últimos años. ${ }^{3}$

Es especialmente notable el proceso de descentralización que la investigación de la comunicación ha experimentado desde mediados de los años ochenta, no sólo por la desaparición de muchos de los núcleos institucionales que operaban en la zona metropolitana de la capital, sino por la instalación de nuevos centros fuera de la ciudad de México. De esta manera, aunque está lejos todavía un equilibrio entre las diversas regiones del país en términos de recursos y producción, las contribuciones provenientes de algunos estados han aumentado considerablemente en cantidad y en calidad, desahogando un poco la presión que se había acumulado sobre los investigadores y los centros de investigación ubicados en la capital, para dar cuenta del panorama comunicacional nacional (Fuentes y Sánchez, 1992: 34).

Finalmente, cabe hacer notar que las prácticas de investigación, especialmente cuando se les califica de científicas, exigen la observación de cierto número de criterios, tanto científico-epistemológicos como ético-políticos, que en conjunto determinan la consistencia y el rigor esperados comunitariamente en la producción de conocimiento, y la pertinencia social del trabajo académico, en cada proyecto y programa concretos. A pesar de que los factores englobados bajo estos dos "marcos" de orientación y evaluación de las prácticas de investigación no son analizados en este trabajo por corresponder al plano cognoscitivo, es conveniente retomar aquí la conclusión del estudio ya citado sobre las perspectivas del campo:

(...) habrá que decir que entre los desafíos y perspectivas de la investigación mexicana de la comunicación en los noventa y el siglo ya próximo, quizá la prioridad estará puesta en las condiciones que definen la profesionalidad de los investigadores: por un lado, la

3 Según las publicaciones incluidas en la Sistematización Documental 1986-1994, en preparación por el autor para actualizar la publicada en 1988 , los tres centros en conjunto contribuyeron con el $23 \%$ de los documentos recopilados. Desglosando un poco más, al CEIC corresponde el 10\%, al Proiicom el $7 \%$ y al programa Cultura el $6 \%$ del total nacional en los ocho años considerados. 
consolidación y ampliación de los apoyos laborales e institucionales necesarios para concentrar la dedicación a las tareas de desarrollo científico y académico; por otro lado, el incremento y reconocimiento de la calificación científica, especialmente en lo que corresponde a la solvencia metodológica de las investigaciones, aspecto que, hasta años muy recientes, ha sido particularmente descuidado.

Creemos que a partir de la consolidación de estas bases, es como podrán superarse los juegos de calificaciones y descalificaciones que han prevalecido al interior y desde el exterior de la comunidad de investigadores de la comunicación y, sobre todo, que podrá avanzarse en el mejor cumplimiento de la función social que, en última instancia, otorga sentido al trabajo científico: la generación de un conocimiento sistemático y riguroso, aplicable a la comprension de la "realidad" comunicacional concreta que vivimos, y al mismo tiempopertinente, útil para su eventual transformación democrática (Fuentes y Sánchez, 1992: 35).

De ahí la intención de explorar empíricamente entre los investigadores las representaciones que se hacen del entorno institucional donde realizan sus prácticas.

\section{Una apreciación de las condiciones para la práctica de la investigación}

Entre el último trimestre de 1992 y el primer semestre de 1993 se aplicó a ochenta investigadores mexicanos de la comunicación un cuestionario diseñado para obtener información empírica directa sobre diversos aspectos y dimensiones relativos a las determinaciones socioculturales del campo académico de la comunicación en México. ${ }^{4}$

4 Para la fase empírica de este proyecto se definieron como sujetos los académicos que, adscritos a determinadas instituciones mexicanas de educación superior, realizan actividades de investigación de la comunicación. Primero se seleccionaron las instituciones, según el siguiente procedimiento: de los más de cien establecimientos educativos donde se estudia la comunicación en el país, se eliminaron aquellos que o bien no contaran con cinco o más académicos "de carrera" (tiempo completo o medio tiempo) o bien hubieran establecido su programa de estudios (por lo general licenciatura) sobre la comunicación después de 1987. El criterio fue que sin personal de adscripción fija o una antigüedad mínima de cinco años, los establecimientos no tendrían capacidad de realizar investigación. Esto recortó el número de instituciones a 36. Un segundo recorte institucional se realizó combinando los dos parámetros anteriores (planta de académicos y antigüedad) con la inclusión de programas de posgrado y la cantidad de publicaciones registradas por sus académicos en la sistematización documental previamente elaborada. De esta manera se seleccionaron cinco instituciones: la UNAM, la UAM-X, la Uia, la UdeG y el ITESO ( 3 públicas y 2 privadas; 3 capitalinas y 2 jaliscienses; todas ellas con cursos de posgrado y múltiples publicaciones de su personal). Se solicitóa los directivos delárea de comunicación de cada una de lascinco instituciones la nominación 
El cuestionario incluyó cuatro instrumentos de recolección de datos, de los cuales sólo se informa aquí de los resultados de uno, el relativo a la apreciación de las condiciones para la práctica de la investigación por parte de los académicos. Los supuestos básicos, inspirados en Bourdieu (1988) y en Giddens (1984), son que la práctica de la investigación académica de la comunicación en México, como toda práctica científico-académica, la realizan sujetos individual o colectivamente abocados al desarrollo de ciertos proyectos de producción de conocimiento, en condiciones concretas, tanto socioculturales como materiales, institucionalmente mediadas, y que los sujetos pueden dar cuenta, a través de sus apreciaciones, de la relación que guardan concretamente las condiciones experimentadas en su propia institución con las que se representan como «ideales» o más favorables.

Mediante una escala tipo Likert se presentó a los sujetos una serie de veinticinco items representativos de condiciones específicas para el trabajo de investigación y se les pidió que calificaran el cumplimiento actual de cada una, en una escala de cinco grados delimitada por los términos "satisfactorio" y "no-satisfactorio". A sus respuestas en la escala de cinco grados se asignaron los siguientes valores:

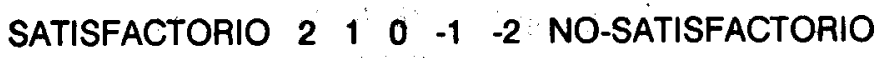

Detodas las respuestas a cadaitem obtenido mediante el paquete SPSS, ${ }^{5}$ el promedio respectivo (media), que puede ser positivo, negativo o cero con límites en 2 y -2, y la desviación estándar. Según el orden descendente de los "grados de satisfacción", las condiciones fueron calificadas por los sujetos como se indica en el cuadro 1.

Mediante la aplicación de la prueba $t$ de Student se buscaron diferencias estadísticamente significativas entre dos grupos de la muestra, según cuatro variables: institución pública o privada; ubicación en la zona metropolitana de la ciudad

[...]de los académicos quéentre sus tareas tuvieran la investigación, y se les hizo llegar a los 73 sujetos así determinados el cuestionario vía las propias instituciones. Además, se incluyeron en la muestra siete sujetos más, adscritos a otras instituciones, para un total de 80 cuestionarios enviados. $\mathrm{Al}$ cierre de la etapa, en mes de julio de 1993, se recuperaron 41 respuestas ( 7 de la UNAM, 12 de la UAM-X, 8 de la Uia, 4 de la UdeG, 4 del ITESO y 6 "individuales").

5 El autor agradece la ayuda de la maestra Margarita Maldonado del Departamento de Psicologia del ITESO para realizar este procesamiento. 


\section{Cuadro 1:}

\section{Calificación de condiciones para la investigación}

\section{Condiciones satisfactorias}

03 "Libertad de acción"

25 "Relación con intereses personales"

22 "Relación con la docencia"

02 "Objetivos bien definidos"

01 "Inserción en programa institucional"

09 "Equipo de computación"

23 "Relación con otros investigadores"

17 "Trascendencia académica"

18 "Reconocimiento institucional"

24 "Relación con necesidades sociales"

19 "Evaluación institucional"

10 "Acervo documental/bibliográfico"

21 "Facilidades de publicación"

12 "Tiempo laboral suficiente"

08 "Infraestructura material"
Media

1.590

1.500

0.923

0.692

0.684

0.667

0.658

0.568

0.500

0.474

0.342

0.333

0.256

0.105

0.026
Desviación estándar

0.677

0.688

1.156

1.173

1.254

1.344

1.236

1.214

1.268

1.156

1.341

1.325

1.446

1.311

1.367

\section{Condiciones no satisfactorias}

16 "Articulación social"

$-0.027$

1.236

07 "Otorgamiento de prioridad"

$-0.028$

1.276

20 "Discusión y orientación"

$-0.077$

1.306

15 "Colaboración interinstituciona!

$-0.108$

1.410

14 "Planificación institucional"

$-0.243$

1.321

06 "Capacitación del personal"

$-0.316$

1.358

13 "Remuneración personal"

$-0.333$

1.420

04 "Financiamiento específico"

$-0.868$

1.212

05 "Personal auxiliar"

$-0.895$

1.311

11 "Presupuesto para gastos"

$-0.895$

1.110

Fuente: cuestionario para investigadores de la comunicación (1993). 
de México u otras ciudades; sexo masculino o femenino del sujeto; estudios de licenciatura del sujeto, en comunicación u otras. Los items en que se encontraron diferencias estadísticamente significativas son indicados en el cuadro 2.

\section{Cuadro 2:}

\section{Diferencias estadísticamente significativas sobre condiciones para la investigación por items}

\section{Según carácter institucional}

Condición

Institución pública Institución privada Prob.t

$n$ media d.s. $n$ media d. $s$.

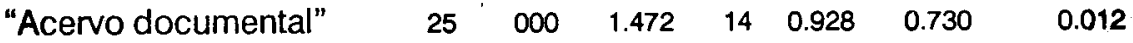

Según ubicación geográfica

Condición

"Financiamiento"
"Personal auxiliar"
"Infraestructura"
"Equipo computación"
"Acervo documental"
"Presupuesto gasios"
"Remuneración"
"Colab. interinstitucional"
"Facilidades publicación"

Ciudad de México Fuera Cd.México Prob.t $n$ media d.s. $n$ media d.s.

$\begin{array}{lllllll}26 & -1.192 & 0.939 & 12 & -0.166 & 1.467 & 0.042 \\ 26 & -1.307 & 1.087 & 12 & 0.000 & 1.348 & 0.009 \\ 27 & -0.481 & 1.282 & 12 & 1.166 & 0.718 & 0.000 \\ 27 & 0.333 & 1.441 & 12 & 1.416 & 0.669 & 0.003 \\ 27 & -0.037 & 1.255 & 12 & 1.166 & 1.115 & 0.006 \\ 26 & -1.269 & 0.919 & 12 & -0.083 & 1.084 & 0.004 \\ 27 & -0.703 & 1.325 & 12 & 0.500 & 1.314 & 0.015 \\ 25 & -0.440 & 1.356 & 12 & 0.583 & 1.311 & 0.039 \\ 27 & -0.148 & 1.379 & 12 & 1.166 & 1.193 & 0.006\end{array}$

Según origen disciplinario

Condición

"Personal auxiliar"

"Infraestructura"

"Presupuesto gastos"

"Árticulación social"

"Rel. necesidades Soc."
Lic. comunicación Otras licenciaturas Prob.t

\begin{tabular}{lllllll}
$n$ & media & d.s. & \multicolumn{1}{c}{$n$} & media & d.s. & \\
28 & -0.607 & 1.397 & 10 & -1.700 & 0.843 & 0.001 \\
29 & 0.379 & 1.321 & 10 & -1.000 & 0.943 & 0.002 \\
28 & -0.642 & 1.096 & 10 & -1.600 & 0.843 & 0.010 \\
28 & 0.214 & 1.228 & 9 & -0.777 & 0.972 & 0.023 \\
28 & 0.750 & 1.041 & 10 & -0.300 & 1.160 & 0.024
\end{tabular}


Para refinar estos análisis estadísticos, las condiciones se agruparon bajo tres tipos generales, descritos en el cuadro 3.

\section{Cuadro 3:}

\section{Agrupación de condiciones para la investigación}

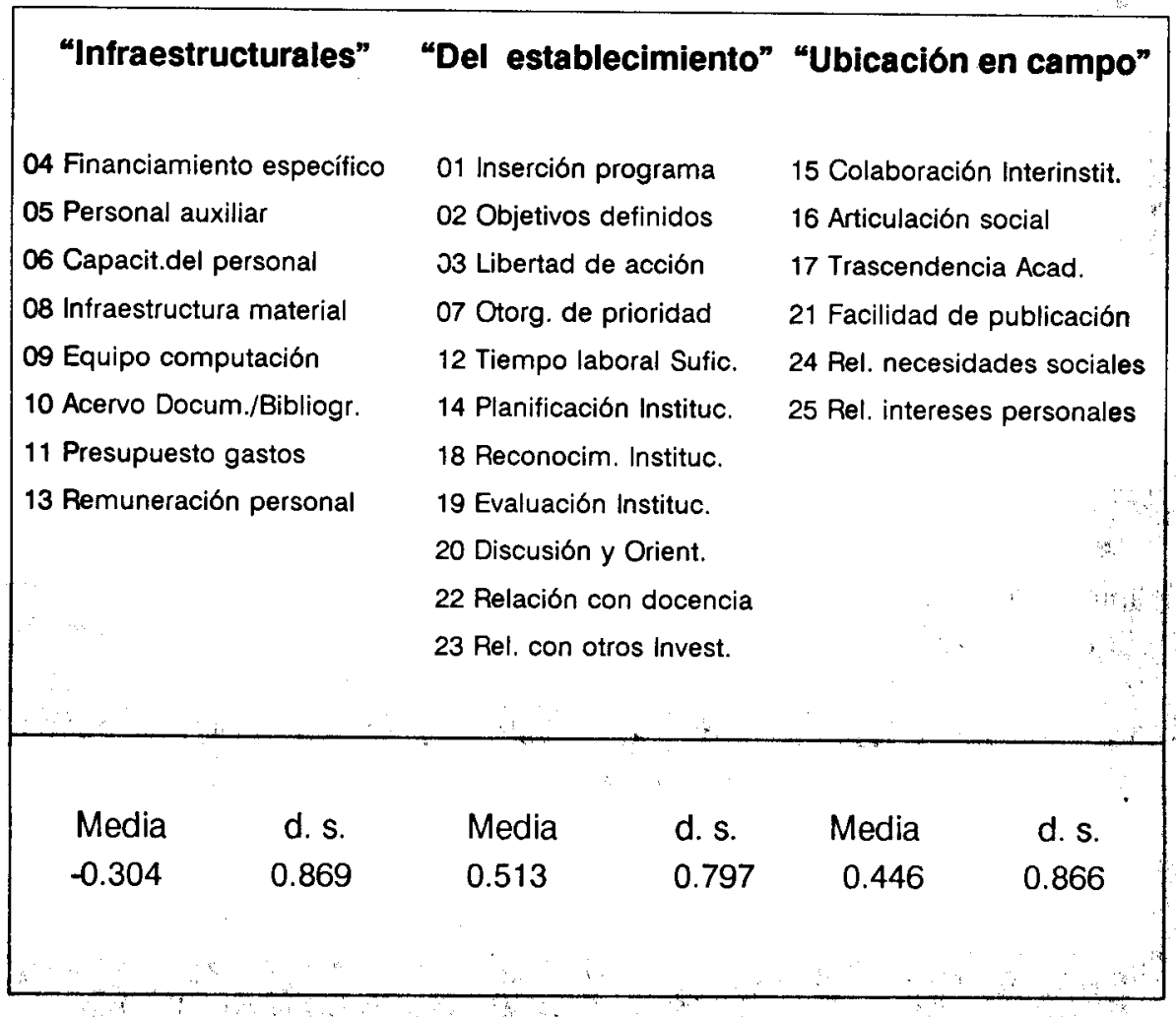

La razón para formar estos tres grupos de "condiciones" tiene que ver directamente con los objetivos del proyecto, pues aunque los tres tipos de condiciones refieren a la inserción del investigador en una institución académica, es necesario distinguir en esa inserción al menos tres dimensiones: la de la dotación de recursos (condiciones infraestructurales), la de las políticas internas (condiciones del establecimiento) y la de las articulaciones extra-institucionales de la investigación (condiciones de ubicación en el campo). 
Utilizando la misma prueba $t$ de Student, se buscaron diferencias estadísticamente significativas en estos tres grupos de items, según las mismas cuatro variables anteriormente señaladas. Dos de ellas dieron por resultado ninguna diferencia estadísticamente significativa: ni el carácter de la institución (pública o privada) ni el sexo del sujeto (masculino o femenino). Los resultados de las otras dos se indican en el cuadro 4.

\section{Cuadro 4:}

Diferencias estadísticamente significativas entre grupos de condiciones para la investigación

\section{Según ubicación geográfica}

\begin{tabular}{lccccccc} 
Gpo. de condiciones & \multicolumn{1}{c}{ Ciudad de México } & \multicolumn{4}{c}{ Fuera Cd. México } & Prob. t \\
& $n$ & media & d.s. & $n$ & media & $d . s$. & \\
"Infraestructurales" & 25 & -0.695 & 0.702 & 12 & 0.510 & 0.573 & 0.000 \\
"Ubicación campo" & 23 & 0.195 & 0.803 & 11 & 0.969 & 0.781 & 0.014
\end{tabular}

\section{Según origen disciplinario}

Gpo.de condiciones Lic. comunicación Otras licenciaturas Prob.t

$\begin{array}{lcccrccc} & n & \text { media } & \text { d. } s . & n & \text { media } & \text { d. s. } & \\ \text { "Infraestructurales" } & 27 & -0.120 & 0.881 & 10 & -0.800 & 0.638 & 0.017 \\ \text { "Ubicación campo" } & 25 & -0.626 & 0.868 & 9 & -0.055 & 0.672 & 0.027\end{array}$

Fuente: Cuestionario para investigadores de la comunicación (1993).

Aunque la pruebat de Student es una herramienta estadística diseñada para detectar diferencias, se consideró que sus resultados pueden también utilizarse para lo opuesto: para captar dónde coinciden las medias correspondientes a dos segmentos de una muestra con respecto a una variable. En el caso del cuestionario cuyos resultados se analizan, es sin duda interesante revisar en qué coinciden los sujetos. Para esto, así como se utilizó una medida de probabilidad igtual o menor a .05 para definir una diferencia estadísticamente significativa, se utiliza una medida de probabilidad igual o mayor a 0.950 para indicar una "identidad estadísticamente significativa". Los resultados se presentan en el cuadro 5. 


\section{Cuadro 5: \\ "Identidades" estadísticamente significativas entre condiciones para la investigación}

\begin{tabular}{|c|c|c|c|c|c|c|c|}
\hline \multicolumn{4}{|c|}{ Según carácter institucional } & \multicolumn{2}{|c|}{ 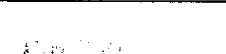 } & \\
\hline \multirow[t]{2}{*}{ Condición } & \multicolumn{3}{|c|}{ Institución pública } & \multicolumn{3}{|c|}{ Institución privada } & \multirow[t]{2}{*}{ Prob. $\boldsymbol{t}_{\text {, }}$} \\
\hline & $n$ & media & d. s. & $n$ & media & d. $s$. & \\
\hline "Financiamiento" & 24 & -0.875 & 1.361 & 14 & -0.857 & 0.949 & 0.962 \\
\hline \multicolumn{8}{|c|}{ Según ubicación geográfica } \\
\hline \multirow[t]{2}{*}{ Condición } & \multicolumn{3}{|c|}{ Ciudad de México } & \multicolumn{3}{|c|}{ Fuera Cd. México } & Prob. $t$ \\
\hline & $n$ & media & d.s. & $n$ & media & d. $s$. & \\
\hline "Eval. Institucional" & 26 & 0.346 & 1.413 & 12 & 0.333 & 1.231 & 0.978 \\
\hline "Rel. Int. personales", & 26 & 1.500 & 0.707 & 12 & 1.500 & 0.674 & 1.000 \\
\hline \multicolumn{8}{|l|}{ Según sexo } \\
\hline \multirow[t]{2}{*}{ Condición } & \multicolumn{3}{|c|}{ Masculino } & \multicolumn{3}{|c|}{ Femenino } & Prob. $\mathbf{t}$ \\
\hline & $n$ & media & d. s. & $n$ & media & d. s. & \\
\hline "Financiamiento" & 21 & -0.857 & 1.276 & 17 & -0.882 & 1.166 & 0.950 \\
\hline "Planif. Institucional" & 20 & -0.250 & 1.209 & 17 & -0.235 & 1.480 & 0.974 \\
\hline "Rel. Int. personales" & 20 & 1.500 & 0.688 & 18 & 1.500 & 0.707 & 1.000 \\
\hline \multicolumn{8}{|c|}{ Según origen disciplinario } \\
\hline \multirow[t]{2}{*}{ Condición } & \multicolumn{3}{|c|}{ Lic. comunicación } & \multicolumn{3}{|c|}{ Otras licenciaturas } & \multirow{2}{*}{ Prob.t } \\
\hline & $n$ & media & d. s. & $n$ & media & d. $s$. & \\
\hline "Prog. institucional" & 29 & 0.689 & 1.312 & 9 & 0.666 & 1.118 & 0.959 \\
\hline "Rel. Int. personales" & 28 & 1.500 & 0.694 & 10 & 1.500 & 0.707 & 1.000 \\
\hline \multicolumn{8}{|c|}{ Entre grupos de condiciones, ubicación geográfica } \\
\hline \multirow[t]{2}{*}{ Gpo. de condiciones } & \multicolumn{3}{|c|}{ Ciudad de México } & \multicolumn{3}{|c|}{ Fuera Cd. México } & \multirow[t]{2}{*}{ Prob.t } \\
\hline & $n$ & media & d. s. & $n$ & media & d. s. & \\
\hline "Del establecimiento" & 23 & 0.505 & 0.799 & 11 & 0.528 & 0.833 & 0.940 \\
\hline
\end{tabular}

Fuente: Cuestionario para investigadores de la comunicación (1993). 
Con vistas a la interpretación de estos resultados en el marco de análisis de la organización social del campo académico de la comunicación en México como una dimensión básica de su institucionalización, habría que subrayar que el promedio general de las apreciaciones es apenas positivo: la calificación del conjunto de sujetos sobre la totalidad de las condiciones es de 0.221 . Por institución, va del 0.600 en la UdeG, 0.380 en el ITESO y 0.220 en la Uia, a 0.020 en la UNAM y -0.130 en la UAM-X. Llama la atención que según los propios investigadores, las "mejores" condiciones generales de trabajo se encuentren en las dos instituciones de Guadalajara, y que el único promedio negativo corresponda a la UAM-X, quizá por el deterioro que sufrieron estas condiciones en los años ochenta y no porque en sí sean malas. ${ }^{6}$ Sin embargo, todas las calificaciones apuntan hacia un mínimo grado de satisfacción.

Trabajando con los "índices" construidos agrupando condiciones, llama de inmediato la atención que el conjunto de investigadores evalúa negativamente las condiciones "infraestructurales" (media $=-0.304$ ). Las diferencias estadísticamente significativas al respecto se dan dividiendo a los sujetos por la ubicación geográfica de suinstitución y por su licenciatura de origen, aunque estas condiciones son peor apreciadas por los investigadores de las universidades públicas que por los de las privadas, y por las mujeres que por los hombres.

La media general de las "condiciones del establecimiento" (media $=0.513$ ) es la menos baja de los tres índices, lo cual puede apuntar hacia una fuerte identificación de los sujetos con sus instituciones. ${ }^{7}$ Ninguna diferencia alcanza a ser estadísticamente significativa a este respecto: las medias son muy parecidas en los distintos subgrupos de sujetos analizados.

Finalmente, las "condiciones de ubicación en el campo" (media $=0.446)$ son apreciadas ligeramente mejor por los académicos de las universidades privadas que por los de las públicas, lo cual no deja de ser sorprendente, y por los hombres que por las mujeres. Sin embargo, las diferencias estadísticamente significativas

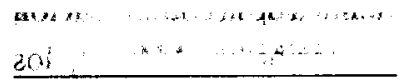

6 Es creencia generalizada en el campo que, a partir de su creación en 1974 y hasta 1982 aproximadamente, las condiciones de trabajo "idcales" para un académico de la comunicación, se encontraban precisamente en la UAM-X. Así loconfirman algunos de los sujetos entrevistados para este proyecto, al mismo tiempo que subrayan, al menos, la "caída salarial" de los ochenta y su "recuperación" mediante estímulos en los noventa.

7 Lo cual correspondería congruentemente al menos a dos datos adicionales: el promedio de antigüedad en sus plazas académicas es de poco más de 12 años para los sujetos de la muestra, y alrededor de la mitad de ellos trabaja en la misma institución donde estudió. 
marcan una mejor apreciación al respecto de los académicos de fuera de la ciudad de México, lo cual también sorprende un poco, y de los investigadores con licenciatura en comunicación.

En general se detecta una oposición muy fuerte entre "satisfacción personal" (nuevo índice construido agregando "relación con intereses personales" con "libertad de acción"), calificada muy alta (media $=1.545$ ) por un extremo y "satisfacción económica" (construido agregando "remuneración personal", "financiamiento específico" y "presupuesto para gastos"), apreciada muy negativamente (media $=-0.698)$ por el otro. Quizá no sorprenda que la "satisfacción personal" tenga una calificaçión tan alta, sin diferencia entre subgrupos; es un poco más sorprendente que sea mayor la "insatisfacción económica" entre quienes trabajan en la ciudad de México, entre quienes no tienen licenciatura en comunicación y entre las mujeres que entre los hombres. ${ }^{8}$

Llama la atención que con respecto a las condiciones institucionales para la práctica de la investigación los sujetos no aporten diferencias estadísticamente significativas entre las universidades públicas y las privadas, excepto en cuanto a la disponibilidad de "acervo documental" en favor de las privadas. Igualmente, que no haya diferencias estadísticamente significativas en las apreciaciones de hombres y de mujeres. En cuanto a las diferencias por licenciatura del sujeto, los comunicadores parecen estar relativamente más conformes con las condiciones que los que estudiaron otra cosa.

Donde sorprende un poco encontrar el mayor número de diferencias estadisticamente significativas es en la división entre los investigadores de la capital y los "de provincia": resulta claro que, en general y en muchos aspectos específicos, los académicos que trabajan fuera de la ciudad de México lo hacen en condiciones - para ellos - más satisfactorias. Este es un dato que requiere mayor análisis, pero que no puede desarrollarse aquí.

En una síntesis provisional, dado que los análisis se encuentran aún en proceso, puede afirmarse quee, además de que la investigación académica de la comunicación se realiza en muy pocas instituciones de educación superior en México, los

8 De estos datos surge una pregunta interesante con respecto a los motivos de permanencia en el campo académico a pesar de la crisis económica de los ochenta. Varios de los sujetos entrevistados aportaron respuestas muy iluminadoras de esa oposición "satisfacción personal-satisfacción económica" y el predominio del primer término sobre el segundo, cuyo análisis no se presenta aquí por estar aún en proceso. 
académicos adscritos a éstas encuentran condiciones apenas mínimamente satisfactorias para su práctica, especialmente en lo referido a infraestructuras, y que parece haber un ingrediente muy fuerte de búsqueda de satisfacción de "intereses personales" entre los investigadores, que les mueve a superar, bajo ciertas circunstancias, las "desfavorables" condiciones que les ofrecen las instituciones."

Por ello, siguiendo a Bourdieu en cuanto a que "el principio de la acción histórica ... no reside en la conciencia ni en las cosas, sino en la relación entre dos estados de lo social, es decir, entre la historia objetivada en las cosas, en forma de instituciones, y la historia encarnada en los cuerpos, en forma de esas disposiciones duraderas que yo llamo habitus" (Bourdieu, 1990: 69-70), el análisis del campo académico de la comunicación en México y sus condiciones institucionales de desarrollo, requiere también la consideración estructural de los factores que determinan su futuro.

\section{El contexto institucional de la polarización universitaria}

Entre los múltiples estudios estructurales del sistema mexicano de educación superior, un ensayo reciente de Casillas y De Garay (1992) resulta particularmente relevante para los propósitos de este trabajo, pues en él se interpretan "los fenómenos asociados con la acelerada expansión del nivel de la educación superior" entre 1960 y 1990 como "contexto de la constitución del cuerpo académico a escala nacional". Este análisis del "periodo más dinámico de la expansión y diferenciación universitaria" en México proporciona entonces un marco imprescindible para el estudio del proceso de profesionalización de los investigadores académicos de la comunicación, y sirve para ubicar el contexto en que se ha desarrollado y expandido

9 No obstante que por la insuficiencia analítica presente no pueden aún aportarse las evidencias conclusivas, puede adelantarse a manera de hipótesis la presencia compartida de múltiples rasgos constitutivos de un habitus específico de los investigadores académicos de la comunicación en México, consistente en un "sistema de disposiciones durables y transferibles que, in tegrando todas las experiencias pasadas, funciona en cada momento como una matriz de percepciones, de apreciacionesy de acciones" (Bourdieu, 1972: 174). Una buena parte de la explicación de las muchas coincidencias detectadas en el discurso de los sujetos (englobables casi todas bajo el rubro "relación con intereses personales") parece provenir de una "cultura generacional" formada en algunas universidades mexicanas entre los estudiantes de la primera mitad de los años setenta: se trataría de la "cultura de la generación post-68", encarnada en sujetos cuyas representaciones y prácticas no coinciden con las de académicos más viejos o más jóvenes. 
Condiciones institucionales para el ejercicio académico de la investigación...

el estudio de la comunicación en las universidades mexicanas. Casillas y De Garay analizan la evolución del sistema mexicano de educación superior, primero en la "época del desarrollo" (1960-1982) y luego "durante el periodo de la crisis" (1982-1990). En síntesis,

En los últimos treinta años, el cambioen la educación superior se diosin la gran reforma, esto es, ocurrió sin un proyecto concebidoy organizado que marcara su rumbo; muchos de los efectos de las políticas educativas fueron "perversos" en el sentido que noestaban previstos o que su efecto agregado se desarrollo en contra de las intencionalidades políticas originales. Fue un proceso "irracional" desde el punto de vista de algunos planificadores, fue el producto de diversos intereses y posiciones que se confrontaron yencontraron peculiares formas de articulación, resultadode la obra de distintos sujetos, de su confrontación y conciliación, fue una síntesis contradictoria interpretada de muy diverso modo por sus protagonistas (Casillas y De Garay, 1992: 14-15).

Durante las tres últimas décadas, el cuerpo académico nacional pasó de poco más de diez mil en 1960 a más de cien mil en 1990, siguiendo la expansión de la matrícula estudiantil, el crecimiento del número de carreras ofrecidas y la multiplicación de las instituciones. Las cifras sobre la expansión de la matrícula en el sistema, sistematizadas por Guevara Niebla y otros en La catástrofe silenciosa, permiten observar "en el último sexenio una caída notable en las tasas de crecimiento de la educación superior. Entre 1970 y 1976 este nivel de estudios creció 112\%; entre 1976 y $1982,67.9 \%$, y entre 1982 y 1988 sólo creció $24.7 \%$. A este fenómeno de contracción relativa corresponde una caída en las tasas de absorción de la demanda, que fue muy variable" (Guevara et al, 1992: 56). Aquí hay que notar también que, aunque los estudiantes de posgrado representan un porcentaje casi insignificante con respecto a los de licenciatura, su crecimiento fue mayor en los años ochenta, como muestra el cuadro 6.

Esta "expansión acelerada" del sistema mexicano de educación superior en los años setenta es muy relevante para nuestro estudio, primero como contexto del crecimiento de la matrícula estudiantil y luego, consecuentemente, de la apertura del mercado académico para muchos jóvenes egresados (De Ibarrola, 1986-1987; Gil Antón, 1990; Valenti, 1990). Fue en esta década, precisamente, cuando se "sentaron las bases" de la institucionalización de la investigación de la comunicación y cuando la mayor parte de los investigadores actuales iniciaron su carrera acadé- 


\section{Cuadro 6:}

\section{Matrícula de la educación superior en México}

\begin{tabular}{|c|c|c|c|c|c|}
\hline Matrícula total & $1970-71$ & $1976-77$ & $1982-83$ & $1988-89$ & $1994-95^{\star}$ \\
\hline Licenciaturas & 284,000 & 548,000 & 989,000 & $1^{\prime} 276,000$ & $1 ' 342,000$ \\
\hline Posgrados & 6,000 & 21,000 & 29,000 & 47,000 & 64,000 \\
\hline$:$ & & & & \multicolumn{2}{|c|}{${ }^{*}$ Tendencia proyectada } \\
\hline
\end{tabular}

(Datos: Guevara et al, 1992)

mica. Aunque hay condiciones diversas antes y después de 1982 en el sistema, hay ciertas constantes en cuanto al mercado académico:

El desarrollo del cuerpo académico durante el periodo 1960-1982 se caracterizó por una clara tendencia hacia la profesionalización. Se generó un tipo profesional nuevo que se dediça de manera central al trabajo académico. Esto quiere decir que la universidad se ha convertidoen el centrode referencia más importante de su desempeño laboral, que vive de la academia y en las instituciones educativas construye su identidad. Por profesionalización entendemos al proceso mediante el cual el trabajo académico es el referente central y la ocupación principal de los académicos contemporáneos (Casillas y De Garay, 1992: 44).

Durante el periodo, la profesionalización de los académicos en México, así entendida, se sustenta en cifras como las siguientes: mientras que en 196589 por ciento de las plazas académicas eran contratadas por horas, este porcentaje se redujo a 75 por ciento en 1980 . Es decir, dentro del espectacular aumento de las plazas académicas totales (14,495 en 1965 y 51,878 en 1980), las de tiempo completo pasaron de 7 a 17 por ciento y las de medio tiempo de 4 a 8 por ciento en esos quince años. Pero en la última década,

El impacto de la crisis económica y de la estrategia gubernamental para enfrentarla, fue devastador para el desarrollo del sistema. Al perder las bases financieras de su sustentación, las dinámicas de expansión y diversificación se frenaron, cerrando un largociclo de crecimiento. El deterioro financierode las instituciones fue impresionante, 
los efectos institucionales de esta caída afectaron principalmente a las comunidades de profesores y trabajadores del sector público, sobre todo si se considera que cerca del $90 \%$ del total del gasto universitario se destina a salarios. (...) Respecto al desarrollo del cuerpo académico, este periodo [1982-1990] resultó paradojico, pues se mantuvo en términos generales la dinámica de crecimiento en el número de plazas (Casillas y De Garay, 1992: 52-54).

Entre 1982 y 1989 se abrieron 26,998 plazas y el "personal de carrera" (tiempos completos y medios) pasó de 26.8 por ciento en 1982 a 32.9 por ciento en 1989 . De manera que, en la escala más general del sistema, uno de cada tres académicos es de carrera. Sin embargo, en el aspecto salarial la crisis fue muy notoria, según una cita textual de Olac Fuentes en Casillas y De Garay (1992: 55):

Hacia 1980 los salarios vigentes en el sector profesionalizado del mercado académico eran relativamente favorables; un profesor joven, con la categoría intermedia de asociado, ganaba entonces entre 6 y 7 veces el salario mínimo y disfrutaba en general de favorables condiciones para hacer una carrera de vida en la academia. El derrumbe fue rápido y sostenido; a principios de 1989 ese mismo profesor ganaba entre 4 y 5 salarios mínimos. Este es un caso privilegiado, que contrasta con el más precario de los maestros profesionales que laboran por hora-clase.

Casillas y De Garay concluyen su ensayo anotando "algunos desafíos de la presente década", que se resumen en los siguientes párrafos:

Si nuestras conjeturas son correctas, para el caso del sistema de educación superior parece claro que la fase de expansión no regulada está agotada, no sólo porque no tiene bases financieras que le permitan sostenerse como hace algunos años, sino también porque los principales sectores sociales demandantes de educación superior han transformado sus relaciones políticas con el gobierno y las instituciones, exigiendo servicios educativos de mayor calidad y eficacia, con lo que la lógica tradicional de funcionamiento gubernamental hacia el sistema de educación superior ha empezado a operar con otros parámetros, menos ligados a la costumbre del llamado intercambio político y, al parecer, más cercana a una perspectiva que pretende una modernización acorde con la visión global de reforma del Estado.

(...) Se perfila una nueva relación entre el Estadoy las universidades. El financiamiento se establecerá a partir de metas pactadas, contratos institucionales de trabajo y la evaluación periódica de resultados. Los objetivos de elevar la calidad y el rendimiento (eficacia y efectividad) se verán acompañados de formas voluntarias de autoevaluación y evaluación externa (1992: 56-57). 
En efecto, en su comparecencia ante la Comisión de Educación de la Cámara de Diputados en marzo de 1992, el subsecretario de Educación Superior e Investigación Científica de la Secretaría de Educación Pública (SEP), Antonio Gago Huguet, presentó un diagnóstico muy documentado del estado de la educación superior en el país, y las "directrices de las líneas de acción prioritarias" que señala el Programa paralaModernización Educativa "para propiciar la transformación de la educación superior":

A. Evaluación de la educación superior. Se plantea la instalación y funcionamiento de seis comisiones nacionales que habrán de llevar a cabo tareas de estudio y promoción de cambios en las áreas del desarrollo institucional. Del trabajo de estas comisiones destaca el de la Comision Nacional de Evaluación de la Educación Superior (Conaeva), debido al impacto que deberá tener en los procesos de reorientación e innovación, no sólo de los centros educativos en particular, sino del sistema global de educación superior.

B. Criterios de financiamiento. Además de la asignación tradicional de recursos públicos, el gobierno federal viene operando una modalidad de financiamiento, basada ya noen criterioscuantitativos comoel tamaño de la matrícula estudiantil o de la planta de personal académico, sino en criterios cualitativos. En esta modalidad, se asignan recursos extraordinarios para proyectos universitarios sólidamente articulados, que resultan de los procesos de autoevaluación, que buscan mejor calidad y que pueden tener impacto estructural en las instituciones.

C. La apertura y vinculación social de las instituciones. Se garantizará una mayor conciencia y confiabilidad en la educación universitaria en la medida en que sean las instituciones educativas y la sociedad civil, a través de los gremios y asociaciones de las distintas disciplinas y profesiones, y mediante decisiones colegiadas, las que determinen los criterios, estándares y parámetros básicos de calidad. Esto se debe a la convicción social, bien fundamentada, de que son los pares quienes pueden conducir de mejor forma el proceso de acreditación profesional.

D. La innovación de las funciones sustantivas. Formar recursos humanos competentes y de alto nivel implica necesariamente producir innovaciones en el enfoque educativo prevaleciente; en los planes y programas de estudio; en las modalidades de aprendizaje estudiantil; en la forma de organizar y llevar a cabo la práctica docente y la investigación; en el quehacer cotidiano, la formación y la organización del personal académico; y en las tareas de extensión. 
E. Descentralización y simplificación administrativa. Deben realizarse en dos ámbitos concretos: en el interior de las propias instituciones y en la relación entre éstas y el gobierno federal. (Gago, 1992: 20-24).

Por lo que toca a la investigación, seguramente el instrumento "modernizador" más importante que ha implantado el Estado es el Sistema Nacional de Investigadores (SNI), instituido en 1984, con los siguientes objetivos:

I Fomentar el desarrollo científico y tecnologico del país fortaleciendo la investigación en cualesquiera de sus ramas y especialidades, a través del apoyo a los investigadores de las instituciones de educación superior y de investigación en México;

II Incrementar el número de investigadores en activo con que cuenta el país, elevando su nivel profesional;

III Estimular la eficiencia y calidad de la investigación;

IV Promover la investigación que se realiza en el pafs, de acuerdocon las prioridades establecidas en el Plan Nacional de Desarrollo;

V Apoyar la formación de grupos de investigación en las entidades federativas del país;

VI Contribuir a la integración de sistemas nacionales de información científica y tecnologica por disciplina, que incrementen y diversifiquen los servicios vigentes actualmente (SNI, 1991: 12).

El Sistema Nacional de Investigadores creció en número de miembros, sostenidamente hasta 1993, cuando disminuyó6 por ciento. De 1396 investigadores admitidos en 1984, el SNI pasó a 6,233 en 1993, distribuidos por categorías, áreas y niveles, como se indica el cuadro 7.

Como puede verse, el SNI establece rigurosamente una distribución piramidal por categorías (candidato/investigador nacional) y niveles. Así, en el área de Ciencias Sociales y Humanidades 29 por ciento de los miembros son candidatos, 50 por ciento investigadores de nivel I, 14 por ciento de nivel II y sólo 7 por ciento de nivel III, es decir, siete puntos porcentuales por debajo de la proporción general en candidatos, cinco por arriba en investigadores de nivel $\mathbf{I}$, uno por debajo en nivel II y casi dos por encima en nivel III. La edad promedio de los miembros del SNI es de 46 años, aunque los investigadores del área de Ciencias Sociales y Humanidades son los de promedio más alto, con 49 años. Entre los miembros totales del SNI el 75 por ciento son hombres, mientras que en el área de Ciencias Sociales y 


\section{Cuadro 7: \\ Miembros del Sistema Nacional de Investigadores \\ por áreas, categorías y niveles}

\begin{tabular}{|lrrrrr|}
\hline & Candidato & Nivel I & Nivel II & Nivel III & TOTAL \\
Físico-matemáticas & 208 & 445 & 174 & 86 & 913 \\
Biología y química & 711 & 855 & 255 & 113 & 1,934 \\
C. Soc. y Human. & 431 & 766 & 205 & 106 & 1,508 \\
Ing. y tecnológicas & 924 & 744 & 163 & 47 & 1,878 \\
TO TAL & 2,274 & 2,810 & 797 & 352 & 6,233 \\
& & & & & \\
\end{tabular}

SEP/Conacyt, 1993: 46

Humanidades la proporción es de 62 por ciento de hombres y 38 por ciento de mujeres, la distribución menos desequilibrada de las cuatro áreas.

Con datos correspondientes a 1992, Yacamán y Alzati (1993: 35) proporcionan la información desglosada más reciente disponible sobre la distribución de los Investigadores Nacionales del área de Ciencias Sociales y Humanidades por disciplina o especialidad. Las cuantitativamente mejor representadas son la Historia (269 investigadores), la Antropología (186), la Sociología (162), la Economía (149) y la Literatura (102). Bajo la denominación "Información", la más cercana a la investigación de la comunicación, se incluye a 8 miembros del SNI. ${ }^{10}$ Tanto el reconocimiento como el estímulo económico (beca complementaria al salario) que representa el SNI para los académicos, alcanzan a muy pocos investigadores

10 La investigación de la comúnicación no ha sido reconocida como una "disciplina" o "especialidad" con identidad propia. Por ello en el SNI, algunos investigadores de la comunicación son ubicados en la categoría "Información" y otros se identifican más bien bajo rubros como "Sociología" o "Antropologia". No obstante esta indistinción, son miembros del Sistema Nacional de Investigadores por lo menos 14 investigadores de la comunicación, la mayor parte de ellos con categoría de candidato o investigador de nivel I. 
mexicanos de la comunicación, aunque su número aumenta cada año, conforme varios de ellos van avanzando en su calificación académica, sobre todo mediante los estudios de posgrado: de los 41 académicos encuestados para el presente proyecto, 10 cuentan con el grado de doctor y diez más están en vías de obtenerlo, casi todos en Sociología, Ciencias Sociales u otra especialidad, pues no hay programas de doctorado en Comunicación en México. Un investigador de la comunicación miembro del SNI, no obstante, advierte que:

Si bien la aparición del SNI en un momento de crisis económica generalizada en el país, y por supuesto de crisis de las universidades públicas, abre una alternativa a algunos investigadores para obtener un merecido reconocimiento social y la posibilidad de seguirse dedicando a sus actividades, para la mayoría de los investigadores y especialmente para la mayoría de quienes investigan dentro de las disciplinas sociales y humanísticas, el SNI no representa la misma alternativa. Esto en parte debido a las peculiaridades de la investigación en estos campos y en parte debido a las tradiciones científicas de los que la realizan. Por el contrario, plantea un enorme desafío: o se juega con las reglas universalistas de excelencia o los investigadores se quedan en la banca, relegados en todo caso a la realización de proyectos marginales, aunque muchas veces heroicos.

Entre estas reglas que se están imponiendo a la práctica investigativa están: grado de doctor, docencia muy productiva, contabilizada en número de cursos impartidos y de tesis concluidas, participación en foros académicos con ponencia y publicaciones con arbitraje, y citas de otros colegas a los trabajos publicados. Estas reglas se han venido haciendo cada vez más rígidas, ya que la exigencia ha aumentado. (...) En este escenario, cada vez más competido, excluyente y sancionado por agentes e instituciones externas, sólo cierto tipo de académicos y ciertas instituciones pueden contar con los recursos necesarios para realizar investigación. Esto supone además de un control estandarizado, discutible en símismo aunque no necesariamente negativo per se, que también hay una concentración de recursos y de campos de estudio, así como de áreas geográficas (Orozco, 1994).

En consecuencia, para tratar de esbozar la relación - tanto la actual como la deseable - entre las "dinámicas internas" de las escuelas, departamentos y centros de investigación en comunicación y el más inmediato de sus contextos, el de las instituciones universitarias donde se insertan, habría que situar con mucha precisión el sentido de las profundas transformaciones que se están operando en México en la mayor parte de las universidades, tanto públicas como privadas, dentro de los procesos sociales de "modernización" y "reforma educativa". No es desmesurado 
caracterizar comopolarización universitaria una de las tendencias que en los últimos años han aparecido en el sistema. Por una parte,

La concentración de la matrícula en algunas entidades e instituciones ha configurado un cuadro desigual en la educación superior mexicana. Por un lado existen centros con una alta densidad estudiantil en los que se ha derrumbado la calidad academica y, por otro, se dan instituciones de baja matrícula que conservan una posicion marginal en cuanto a atención de parte de las autoridades educativas (Guevara et al, 1992: 59).

Pero al mismo tiempo, conforme se diluyen algunas de las diferencias que en décadas anteriores opusieron a las instituciones públicas y privadas, se establece una nueva polarización: entre aquellas instituciones que pueden hacerse reconocer, en algunos programas, como centros "de excelencia" y las que comienzan a perder las condiciones mismas de viabilidad, por no poder alcanzar reconocimientos oficiales "de calidad" como los otorgados por la SEP y Conacyt. Probablemente por ello, al margen de muchas de las acciones que se emprendan en las escuelas de comunicación, el futuro del campo académico será redefinido en los noventa desde las reformulaciones de la articulación universidad-sociedad que prevalezcan en cada institución donde operan, y las condiciones para la práctica de la investigación dependerán en cada vez mayor medida de ellas.

En este contexto, la revisión de las condiciones institucionales en que se han desarrollado las prácticas de investigación académica de la comunicación en México, permite fijar algunos parámetros a partir de los cuales podrían evaluarse - y apoyarse más decididamente - las posibilidades de desarrollo de este campo en el país. Los datos expuestos en este trabajo ilustran la tensión entre las precarias facilidades con que cuentan estas actividades en algunas universidades - en la mayoría, son de plano inexistentes - y los aportes personales con que los investigadores sostienen sus proyectos - los que en mayor medida coinciden con sus intereses.

Como se ha visto, las condiciones "infraestructurales" son las que presentan las mayores insuficiencias, desde el punto de vista de los propios investigadores, como ha sido el caso al menos en los últimos veinte años. La mayor parte de estas condiciones "infraestructurales" (Véase cuadro 3), tiene que ver directamente con asignaciones presupuestales y dotación de recursos, que las instituciones no parecen haber estado en posición de suministrar en las cantidades necesarias para 
impulsar el desarrollo del campo, aunque los apoyos prestados a los académicos sean más positivamente reconocidos en otros aspectos.

De esta manera, puede concluirse que la institucionalización de la investigación académica de la comunicación en México podrá seguirse sosteniendo a corto plazo de manera análoga a como lo ha hecho en la última década, pero que su futuro - consolidación y crecimiento - dependerá en buena medida de los insumos económicos que puedan invertirse en algunas instituciones para que, a mediano plazo, los investigadores formados puedan continuar trabajando y, sobre todo, para que nuevos académicos, más jóvenes, encuentren condiciones atractivas para incorporarse a las actividades científicas.

\section{Referencias bibliográficas}

BENTTEZ ZENTENO Raúl (1987): Las ciencias sociales en México. México: Comecso/Conacyt.

BoURdiEU Pierre (1972): Esquisse d'une Théorie de la Practique. Geneve: Droz. (1988): Homo Academicus. California: Stanford University Press. (1990): Sociologia y cultura. México: Grijalbo/CNCA, Los Noventa.

BRUNNER José Joaquín (1990): Educación superior en América Latina. Cambios y desafios. Chile: Fondo de Cultura Económica.

CASILLAS Miguel Angely Adrián DEGARAY (1992): "El contextode la constitución del cuerpo académico en la educación superior 1960-1990", En Manuel GIL ANTÓN et al, Académicos, un botón de muestra. México: UAM-Azcapotzalco.

DE IBARROla María (1986-1987): "La formación de investigadores en México. Invitación al debate" (2 partes). Avance y Perspectiva Núms. 29 y 33, pp. 3-21 y 3-37. México: Cinvestav. 
FUENTES NAVARRo Raúl (1988): La investigación de comunicación en México. Sistematización documental 1956-1986. México: Ediciones de Comunicación.

(1991): La comunidad desapercibida. Investigación e investigadores de la comunicación en México. Guadalajara: ITESO/Coneicc.

— (1993): "Para la memoria de la investigación mexicana en ciencias de la comunicación: una charla retrospectiva con Josep Rota". Umbral XXI Núm. 12, pp. 21-28. México: Universidad Iberoamericana.

- yEnrique E. SÁNCHEZ R. (1989): Algunas condiciones para la investigación científica de la comunicación en México. Guadalajara: ITESO, Huella Núm. 17.

- y Enrique E. SÁNCHEZ R. (1992): "Investigación sobre comunicación en México: los retos de la institucionalización". Cuademos de conunicación y prácticas sociales Núm. 3, pp. 11-38. México: Proiicom, Universidad Iberoamericana.

GaGo Huguet Antonio (1992): "Ejes de la reforma: calidad y pertinencia". Universidad futura Vol. 4, Núm. 10, México.

GIDDENS Anthony (1984): The Constitution of Society. Outline of the Theory of Structuration. Berkeley \& Los Angeles: University of California Press.

GIL ANTón Manuel (1990): "El mercado académico en México". Umbral XXI Núm. 3, pp. 2-11. México: Universidad Iberoamericana.

GuEVARA NIEBLA Gilberto et al (1992): La catástrofe silenciosa. México: Fondo de Cultura Económica. 
JARA Elf́as J. Rubén (1981): "Información básica sobre la investigación de la comunicación en México: documentos, instituciones, publicaciones, investigadores, y un análisis del estado actual de la disciplina". Comunicación, algunos temas Año 1, Núms. 2-3-4, México: Cenapro/Armo.

Orozco GómEz Guillermo (1994): "Desafíos de la investigación de la comunicación a fin de milenio: reflexiones desde la práctica". Ponencia en la reunión conmemorativa del XV aniversario de la Asociación Mexicana de Investigadores de la Comunicación (AMIC), México.

ROTA Josep (1974): "Remarks on Journalism Education and Research in the Americas". Mass Communication in Mexico, Proceedings of the March 11-15 Seminar in Mexico City. pp. 56-57. México: Association for Education in Journalism/Universidad Iberoamericana.

SÁNCHEZ RuIZ Enrique E. (comp.) (1988): La investigación de la comunicación en México. Logros, retosy perspectivas. México: Ediciones de Comunicación/Universidad de Guadalajara.

SEP/Conacyt (1993): Indicadores de actividades científicas y tecnológicas 1993. México: SEP/Conacyt.

Sistema NACional DE InVESTigadores (1991): Directorio 1990. México: SEP/SNI/Academia de la Investigación Científica.

VALENTI NIGRINI Giovanna (1990): "Tendencias de la institucionalización y la profesionalización de las ciencias sociales en México". En Francisco José PAOLI (coord.) Desarrollo y organización de las ciencias sociales en México, pp.431-470. México: CIIH UNAM/M. A. Porrúa. 
YACAMÁn Miguel José y Fausto AlZati ARAIZA (1993): "El perfil del SNI y los posgrados de excelencia en México”. Ciencia y Desarrollo Vol. XIX, Núm. 109 (nueva época), pp. 28-46. México: Conacyt. 